\title{
El desarrollo de la Ciencia Política EN URUGUAY
}

\section{Daniel Buquet}

dbuquet@gmail.com

Instituto de Ciencia Política

Facultad de Ciencias Sociales

Universidad de la República

En el artículo se examina cómo se ha consolidado la Ciencia Política en Uruguay tanto en el espacio académico como en el ámbito profesional. Se señala que el desarrollo de la disciplina es tardío, en relación al mundo y a los países más importantes de América Latina, ya que se inicia posterior a la transición a la democracia del año 1985, no obstante ello ha alcanzado un prestigio creciente. Se argumenta que aun cuando existen aspectos en los cuales es posible acrecentar los indicadores, la Ciencia Política uruguaya cuenta con un plantel de investigadores que ostenta buenos niveles de formación y es productivo en términos generales, tanto en cantidad como en calidad.

Palabras Clave: Ciencia Política, publicaciones, docencia, investigación, Uruguay.

\section{The Development of Political Science in URUGUAY}

The article examines how political science has consolidated in Uruguay, both in the academic and professional spheres. Given the discipline's late development in comparison to the rest of the world and the major Latin American countries, having begun following the transition to democracy in 1985, the discipline has attained increasing prestige. It is argued that although there are indicators which may be improved, Uruguayan political science includes a cadre of well prepared researchers who have been productive both in terms of quality and quantity.

Keywords: Political Science, publications, teaching, research, Uruguay. 
A pesar de que Uruguay fue un país destacado en el plano educativo y cultural durante la primera mitad del siglo XX, y que contó tempranamente con un sofisticado sistema universitario, las ciencias sociales tuvieron un desarrollo tardío en el país (De Sierra, 2005). La ciencia política, lejos de ser la excepción, parece más bien la hija menor de un matrimonio envejecido. La disciplina adquirió estatus universitario por primera vez a partir de la creación de la cátedra de ciencia política en la Facultad de Derecho en 1957 a cargo de Alberto Ramón Real, quien desarrollaba un enfoque muy próximo al derecho público (Garcé, 2005). Sin embargo, ya existía una cátedra de sociología desde 1915 y en 1958 se creó el Instituto de Ciencias Sociales dentro de la Facultad de Derecho, ámbito destinado a la investigación, que se dedicaría casi exclusivamente a la sociología, aunque incluyendo entre sus objetos de estudio a la política con enfoques de sociología política. La economía también cobró densidad académica durante los años 50 y 60 con la creación del Instituto de Economía en la Facultad de Ciencias Económicas. En cambio, la ciencia política debió esperar la finalización de la dictadura para disponer de un espacio académico propio desde el cual pudiera desarrollarse, de forma similar a lo ocurrido en Argentina y Chile, aunque en esos países la disciplina ya había comenzado a echar raíces a fines de la década de 1960 (Altman, 2005).

Se ha explicado el desarrollo tardío de las ciencias sociales en Uruguay como consecuencia del conformismo generado por el éxito relativo del país en términos comparativos con la región, mostrando como evidencia que no se realizó ningún censo en el país entre 1908 y 1963 (De Sierra, 2005). Luego, se explica el interés que despertó la economía en esos años como consecuencia del fracaso del modelo de desarrollo basado en la sustitución de importaciones que empezó a manifestarse tempranamente en la década de 1950. Asimismo, también se explica el interés que generó la sociología a partir de la magnitud que adquirieron los conflictos y movilizaciones sociales durante la década de 1960. En ese contexto parece razonable que no hubiera un espacio relevante para la ciencia política, especialmente porque en las ciencias sociales de la época predominaban enfoques estructuralistas que adjudicaban a lo político la condición de epifenómeno.

Posteriormente, la dictadura truncó en buena medida el desarrollo que las ciencias sociales venían alcanzando durante la década de 1960, a semejanza de lo ocurrido en los otros dos países del cono sur de América, Argentina y Chile (Altman, 2005). Luego del golpe de estado en 1973, la mayor parte del personal académico fue expulsado de la universidad, ya que tenían visiones y actitudes opuestas al régimen y la enseñanza de las ciencias sociales fue lisa y llanamente eliminada de las aulas. De 
todas formas, quienes no marcharon al exilio se fueron reorganizando en centros privados de investigación que, gracias a la obtención de fondos externos, mantuvieron el cultivo y también la formación de recursos humanos en las ciencias sociales (De Sierra, 2005) ${ }^{1}$. Una vez restablecida la democracia en el país, con exilados que regresaron y académicos que participaron en los centros privados se formó el núcleo fundacional de la ciencia política uruguaya.

\section{El Instituto de Ciencia Política}

Cualquier descripción de la evolución de la ciencia política en Uruguay debe dedicar un espacio central a la creación y desarrollo del Instituto de Ciencia Política (ICP), por tratarse del ámbito desde el cual se posibilitó el cultivo académico de la disciplina de forma orgánica y sistemática. El Instituto de Ciencia Política se fue conformando en un proceso gradual entre los años 1985 y 1988 en la Facultad de Derecho y Ciencias Sociales de la Universidad de la República (UdelaR). Allí se constituyó el núcleo fundacional de la disciplina a partir de académicos formados en ciencia política en el exterior y otros formados en otras disciplinas en Uruguay. En 1985 se otorga estatuto formal al ICP que, al igual que otros institutos de la Facultad de Derecho, está conformado por los docentes de la materia para realizar actividades de investigación, aunque sin un financiamiento específico. Luego se designa como su primer director al Dr. Julio Barreiro, abogado con una larga trayectoria de militancia cristiana quien se había incorporado en 1971 a la cátedra de Ciencia Política y participó en ese mismo año de la creación de la cátedra de Historia de las Ideas en la misma Facultad (Instituto de Historia de las Ideas, 2007).

El núcleo inicial de docentes estaba constituido fundamentalmente por abogados y profesores de historia y una de sus primeras actividades académicas -fuera de la enseñanza- fue la creación de la Revista Uruguaya de Ciencia Política (RUCP) en 1987, que continúa siendo hasta hoy la principal publicación del ICP. Pero el Instituto cobra verdadero impulso en 1988 (Pérez Antón, 1992) a partir de la designación de Jorge Lanzaro como director, el único de sus integrantes que contaba en ese momento con un título de doctorado en ciencia política. Poco después la Facultad asignó horas de investigación a un grupo de docentes que no llegaba a la decena,

Entre los centros privados que cultivaron las ciencias sociales durante la dictadura merecen destacarse el Centro de Informaciones y Estudios del Uruguay (CIESU), el Centro de Investigaciones Económicas (CINVE) y el Centro Latinoamericano de Economía Humana (CLAEH). Todos ellos mantienen actividades en la actualidad. 
creando la primera partida presupuestal destinada a la investigación politológica. En este nuevo grupo se incorporan algunos académicos que habían estudiado ciencia política en el exterior y otros con distintas formaciones en ciencias sociales y humanidades. Ese año también se creó la licenciatura en Ciencia Política que iniciaría sus cursos el año siguiente. Finalmente, como conclusión de la fase fundacional, el ICP se incorpora en 1990 a la recién creada Facultad de Ciencias Sociales (FCS) como una de sus cinco unidades académicas ${ }^{2}$. Esta inclusión, con el consiguiente refuerzo de sus recursos presupuestales, permitió que a lo largo de la década de 1990 se ampliara la planta académica con la incorporación de nuevos docentes -algunos de los cuales formados en la propia licenciatura-y que se desarrollaran diversas áreas y programas de investigación. Esta segunda fase de consolidación del Instituto culmina con la creación de la Maestría en Ciencia Política en 1997. Al finalizar el siglo pasado el Instituto ya contaba con un plantel docente y una estructura académica muy similar al actual.

A lo largo de todo este período el papel de Jorge Lanzaro fue determinante, ya que lideró todo el proceso, desde la creación del Instituto hasta su consolidación como departamento de la Facultad de Ciencias Sociales, con cursos disciplinarios de grado y posgrado y un amplio cuerpo académico dedicado a la investigación. El período siguiente, que abarca hasta nuestros días, ha estado signado por la rotación en la dirección del Instituto $^{3}$ y avances importantes en cuanto a la calidad de sus actividades. En primer lugar, durante la primera década del presente siglo, la mayor parte del cuerpo de investigadores del ICP obtuvo su doctorado. De una planta de alrededor de 30 investigadores, 19 tienen título de doctorado y, de ellos, 14 lo obtuvieron entre 2000 y 2011 (Buquet, 2011). Por otra parte, el ICP creó su propio doctorado en el año 2005, del que egresaron dos de sus investigadores y que otros cuatro están cursando.

Asimismo otros cinco investigadores del instituto, los más jóvenes, están haciendo sus doctorados en el exterior (Buquet, 2011). Por último la RUCP ha logrado regularizar su periodicidad anual y ha procurado cumplir con estándares internacionales para publicaciones científicas. En ese sentido se estableció a partir de 2006 el sistema de arbitraje y la revista fue incluida en diversos índices internacionales de publicaciones (SCielo, EBSCO, Redalyc y Latindex), logrando situarse como referente, al

2 Las otras unidades académicas de la FCS son los departamentos de Economía, Sociología y Trabajo Social y la Unidad Multidisciplinaria.

3 Gerardo Caetano fue director entre 2000 y 2005, luego Constanza Moreira de 2005 hasta 2008 y finalmente Pedro Narbondo hasta la actualidad. 
menos en el ámbito regional (Buquet, 2011). En definitiva el Instituto tiene una planta docente numerosa que se encuentra organizada en Áreas, Programas y Grupos de investigación que muestran niveles importantes de actividad en función de diversos proyectos de investigación registrados y la difusión de sus resultados a través de numerosas publicaciones. Estos avances en calidad están a tono con las tendencias internacionales en la materia y fueron claramente incentivados por la creación del Sistema Nacional de Investigadores en 2005, al que pertenece la casi totalidad del plantel, aunque el ICP está aún lejos de ostentar una posición de liderazgo en la región (Altman, 2011).

\section{TEMAS Y ENFOQUES DE LA CIENCIA POLÍTICA URUGUAYA}

Los temas y enfoques de la incipiente ciencia política uruguaya son subsidiarios de la formación y los estilos académicos del equipo fundacional. Así, la historia política, la teoría política y la sociología política constituyeron los insumos que conformaron los primeros cursos y productos de la disciplina. Pero también la coyuntura política determinó los intereses fundamentales de la academia e incorporó los primeros componentes propiamente politológicos a la disciplina. Los trabajos sobre la transición a la democracia y la búsqueda de explicaciones para su derrumbe, concentraron el interés académico. En un primer momento se adoptó, por un lado, una visión favorable al parlamentarismo como reflejo de la fuerte influencia que ejercieron los trabajos pioneros de Juan Linz y, por otro, una perspectiva crítica sobre los partidos políticos y el sistema electoral uruguayo.

En ese contexto el sistema de partidos se ubicó en el centro de atención y el debate giró hacia una revalorización de su actuación y su rol en la sociedad. A partir de una mirada elaborada desde la historia política se elaboró una caracterización que pretendía dar cuenta de las peculiaridades del sistema político uruguayo al que se propuso denominar como "partidocracia" (Caetano, Rilla y Pérez, 1987) ${ }^{4}$. Durante algo más de una década la academia uruguaya desarrolló un intenso debate entre quienes cuestionaban, por un lado, y quienes defendían, por otro, a los partidos políticos y el marco institucional dentro del que actuaban. Varias de las publicaciones

4 El uso de este término es una medida del incipiente desarrollo de la disciplina y su consecuente parroquialismo (Chasquetti, 2010), ya que se acuñó sin considerar su utilización generalizada como una deformación de la democracia. Por ejemplo, según Sartori, una partidocracia es "una tiranía de partido, en la que el centro real del poder se ha desplazado, y concentrado, del gobierno y el Parlamento, a, y en, los órganos de dirección de los partidos” (1994:51-52). 
al respecto en esos primeros años surgieron de seminarios que se organizaban para discutir la necesidad de una reforma política o, particularmente, electoral. El interés prácticamente excluyente que concitaron los partidos uruguayos como objeto de estudio, por ser causantes de la caída de la democracia y responsables de su reconstrucción, queda de manifiesto a través de la primera publicación del ICP fuera de la revista. Se trató de la compilación de trabajos presentados en un seminario que tuvo por título "Los partidos de cara al 90" (ICP, 1989).

El interés por los partidos permite también verificar que la ciencia política propiamente dicha surge luego de la dictadura. Una bibliografía sobre partidos políticos que pretendió ser exhaustiva (Caetano, Rilla, Mieres y Pérez 1992) incluye 261 referencias en la materia. De esas obras sólo 43 (16,5\%) fueron publicadas antes del golpe de estado o durante la primera fase de la dictadura, antes que comenzara a salir a luz la producción de los centros privados de investigación. La mayor parte de estos últimos textos corresponden a historia política y una cuarta parte a la incipiente sociología política que se desarrollaba desde el Instituto de Ciencias Sociales. Entre los demás sólo uno de ellos merecería la clasificación de politológico, el ensayo que Real de Azúa dedicó a los partidos políticos uruguayos en 1971 (Real 1988) quien merecidamente ha recibido el título de precursor o pionero -también fundador- de la ciencia política uruguaya (Pérez Antón, 1992; Instituto de Ciencia Política, 2002).

Tomando distancia de los partidos políticos, también puede apreciarse la evolución de los temas y enfoques de la ciencia política en Uruguay a través de los autores de referencia que aparecen en las bibliografias de los artículos publicados por la RUCP. En una primera fase (1987-1995) los autores más citados son Giovanni Sartori, Guillermo O’Donnell, Phillip Schmitter, Juan Linz, Arend Lijphart, Norberto Bobbio y Dieter Nohlen (Rocha, 2012). De esta forma se confirma que la democracia (transiciones y consolidación), los partidos políticos y el marco normativo (régimen de gobierno y sistema electoral) constituyen los intereses fundamentales de la academia. Luego de 1995 esos autores son desplazados por Scott Mainwaring, Mathew Shugart, Gary Cox y Douglas North, acompañados por una mayor presencia de autores nacionales (Rocha, 2012). De esta forma se observa que el neo institucionalismo y los estudios comparados que utilizan métodos cuantitativos cobran mayor relevancia a la vez que la disciplina desarrolla una acumulación propia.

Asimismo, la ciencia política uruguaya fue desarrollando durante la década de 1990 otra área temática que ha devenido en central a nuestros días: el estudio de las políticas públicas. Esta cuestión estuvo relativamente ausente en los primeros años y fue cobrando fuerza a medida que ciertos nudos problemáticos se iban colocando en 
la agenda pública. Así, la reforma del estado, las políticas sociales y, en particular, las políticas educativas han venido concitando creciente atención dentro de la academia y, en los últimos años, buena parte de la producción se ocupa de esos temas. De todas formas, el interés por los partidos, las elecciones y los procesos de gobierno parecen seguir constituyendo el foco principal de las investigaciones. Una revisión de los artículos publicados en la RUCP, arroja que algo más de la mitad de los artículos refiere a dichos temas, mientras que sólo una tercera parte se relaciona con cuestiones de Estado y políticas públicas, aunque la relación se invierte si se consideran los documentos de trabajo publicados por el ICP (Rocha, 2012). Asimismo se verifica un predominio del interés por la politics sobre las policies a partir de una clasificación más detallada de los temas incluidos en los artículos de la RUCP utilizando una serie de palabras clave. Los artículos descritos con los términos democracia, gobierno, partidos, elecciones, presidencialismo/parlamentarismo, opinión pública, parlamento, coaliciones y fracciones llegan casi al 50\% del total de los publicados en la revista, mientras que los que refieren a Estado, políticas públicas, educación,

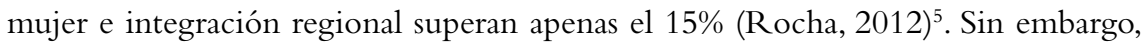
este mayor interés no debe asociarse necesariamente a que la mayor parte de los investigadores o del tiempo de investigación se dedique a esas cuestiones, ya que la producción en el área de políticas públicas ha estado destinada en una importante proporción a publicaciones institucionales de distintos organismos públicos a través de convenios con el ICP y otras instituciones académicas.

Finalmente, también es posible apreciar una evolución en cuanto a las estrategias de abordaje de las diferentes cuestiones, donde se observa un incremento del uso de datos cuantitativos. Sólo una tercera parte de los artículos publicados hasta el año 2000 en la RUCP incluía al menos una tabla o cuadro con números, en tanto que esa proporción se incrementó hasta el 70\% de los artículos publicados posteriormente (Rocha, 2012). Esto no significa que durante las primeras épocas predominaran las técnicas cualitativas, ya que en el total de los artículos de la RUCP menos de un 15\% utiliza entrevistas (Rocha, 2012), sino que confirma que en el periodo inicial predominaba más bien un estilo teórico-ensayístico. Algo similar puede decirse acerca del uso del método comparativo, cuya presencia muestra un leve incremento en los artículos de la RUCP y también en las monografias de grado y tesis de maestría en ciencia política realizadas en la Facultad de Ciencias Sociales (Chasquetti, 2010)

La clasificación desarrollada por Rocha (2012) no permite atribuir a alguna de las dos áreas los artículos clasificados con palabras clave como reforma, izquierda o instituciones, que suman un $32 \%$. 


\section{LA ENSEÑANZA DE LA CIENCIA POLÍTICA EN URUGUAY}

La enseñanza de la ciencia política, iniciada -como se dijo, en 1989- dentro de la Facultad de Derecho, ha sido el principal proveedor de recursos humanos para la disciplina en Uruguay, tanto de investigadores como de profesionales. La licenciatura de ciencia política de la FCS es la única que proporciona una formación integral en la disciplina a nivel de grado, aunque también la Universidad Católica del Uruguay otorga el título de Licenciado en Ciencia Política a partir de una Licenciatura en Ciencias Sociales Aplicadas ${ }^{6}$, que admite la especialización en ciencia política a través de algunos cursos específicos que se toman durante los dos últimos años. Estos son los únicos casos de formación de grado en ciencia política en Uruguay.

Los planes de estudio de ambas licenciaturas se modificaron en dos oportunidades. La licenciatura de la FCS adoptó su plan original de 1988 que fue modificado en 1992, una vez que estaba en funcionamiento la nueva facultad, como consecuencia del interés de unificar algunos criterios para las tres licenciaturas que se impartían entonces $^{7}$. En esa ocasión, las principales modificaciones fueron la reducción de la duración de la carrera, que pasó a dictarse en ocho semestres en lugar de once, como la mayoría de las licenciaturas, y la creación de un ciclo básico común para las tres carreras, que se dictaba durante el primer año. Fuera de estas dos cuestiones, sólo vale la pena agregar la incorporación de cursos de metodología, que se sumaron a los de matemática y estadística del nuevo ciclo básico, favoreciendo la visión más empírica y menos teórica que comenzaba a gestarse dentro de la disciplina. Luego se volvió a modificar el plan de estudios en 2008, respondiendo nuevamente a un proceso general de la FCS que, esta vez, buscaba una mayor integración de las carreras, al tiempo que currículos más flexibles. El nuevo plan de estudios incluyó la creditización de los cursos, la creación de módulos temáticos que incluyen más de una asignatura y un incremento de las materias optativas que pueden seleccionar libremente los estudiantes, estimulando la creación de cursos que sirvan a más de una carrera.

6 Así se llamó desde su inicio en 1991 hasta 2005. Luego pasó a llamarse simplemente Licenciatura en Ciencias Sociales.

7 Además de ciencia política, en la FCS se dictan las licenciaturas de Sociología y de Trabajo Social desde el inicio y desde 2008 se agregó una Licenciatura en Desarrollo. 
En cuanto a los contenidos, esta vez, además de incrementar nuevamente la carga de asignaturas metodológicas, se diseñó un módulo completo dedicado a Estado y Políticas Públicas, cuestión que en los planes anteriores se trataba exclusivamente en una única materia, lo que confirma la aparición tardía y el interés creciente por esta temática. Por su parte, los cambios en los planes de estudios en la UCUDAL se realizaron en 1997 y 2005 y también se orientaron a incrementar la formación metodológica y el espacio destinado a las políticas públicas.

Aunque en otros países lo normal es que exista un número considerable de carreras de ciencia política, en Uruguay esta oferta restringida parece ser más que suficiente. Una medida al respecto está constituida por la demanda, evaluada por el número de estudiantes que ingresan a la carrera en la Facultad de Ciencias Sociales. Este dato es un buen estimador de la demanda total, ya que el ingreso a la carrera es libre para todos los estudiantes que hayan terminado sus estudios secundarios (no existe examen de ingreso ni proceso alguno de selección) y además gratuito, por lo que no hay restricciones de ninguna especie ${ }^{8}$.

En el siguiente gráfico se puede apreciar cómo hubo un proceso creciente de ingresos a la carrera de ciencia política hasta el año 2005 y luego un proceso de estabilización en torno a 170 por año. Por lo tanto podemos suponer que la demanda por realizar estudios de ciencia política se ha estabilizado. De todas formas la información no puede considerarse concluyente, por cuanto en el año 2009 se puso en práctica una reforma del plan de estudios que estableció que la elección de la carrera se realiza en el segundo año y no al ingresar a la facultad. Esa es la razón por la que el año 2009 aparece sin información y los números de los años siguientes son significativamente inferiores, ya que se trata de estudiantes que culminaron el primer año de estudios. Por lo tanto no podemos saber cuántos estudiantes de los que ingresaron en esos años hubieran elegido la carrera de ciencia política al momento de inscribirse en la facultad, aunque nada sugiere que la tendencia creciente se hubiera retomado.

\footnotetext{
En realidad, el lugar de residencia puede ser una restricción importante, ya que la carrera se imparte sólo en Montevideo. De todas formas esto es válido para casi todas las carreras universitarias y existe una tradición ancestral de que los habitantes del interior que desean cursar una carrera universitaria se trasladen a Montevideo.
} 


\section{Gráfico No 1}

Ingresos y egresos de la Licenciatura de Ciencia Política (FCS)

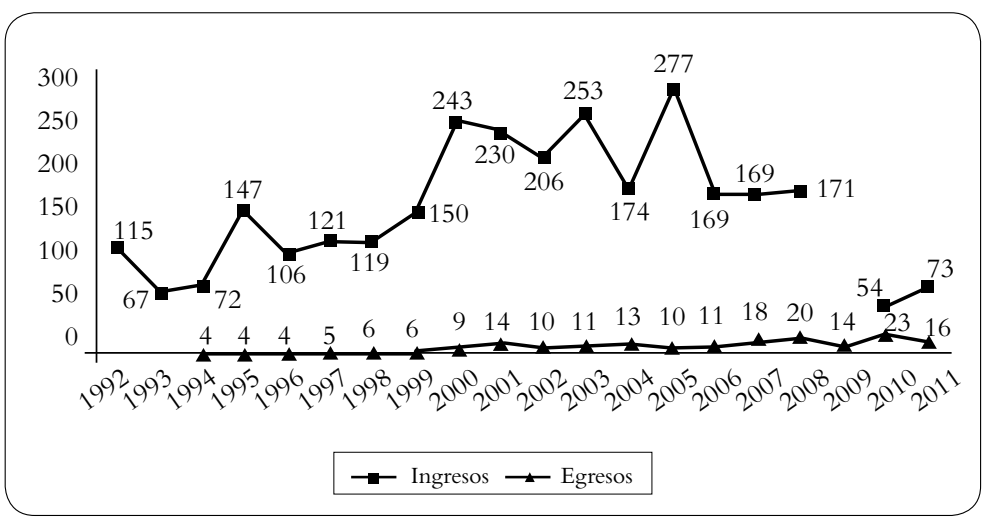

Fuente: elaboración propia en base a datos proporcionados por la Unidad de Apoyo a la Enseñanza (FCS)

En cambio, la modificación del sistema de inscripción a las carreras de la facultad permite apreciar otra característica, que obedece y verifica que el sistema de ingreso es completamente libre, ya que luego de transcurrido un año, la deserción es extraordinariamente alta. Los valores en torno a 200 ingresos por año son en realidad una demanda "inflada", en la medida en que no parece tratarse en su totalidad de personas completamente decididas a estudiar ciencia política, sino que una proporción muy importante de ellos lo toma más bien como una opción a experimentar y que luego descartan. Precisamente en ese sentido se puede apreciar la enorme diferencia entre ingresos y egresos que aparece en el gráfico.

Más allá de que el número de egresos muestra una tendencia creciente que no se corta en el año 2005, hoy por hoy estamos hablando de una veintena de egresados por año, que podría responder a la demanda efectiva de la carrera durante la primera mitad de la década de 2000, es decir de las personas que no sólo deseaban estudiar ciencia política sino que estuvieron dispuestas a realizar el esfuerzo por culminar la carrera. A esta demanda efectiva habría que agregar los egresos de la carrera de UCUDAL, que suman 25 desde los primeros que se produjeron en 1998, es decir un promedio que no alcanza los dos por año y tampoco muestra una tendencia creciente $^{9}$. El país cuenta entonces con algo más de 200 licenciados en ciencia política. 
Por otra parte, la formación en ciencia política a nivel de posgrado comenzó en 1997 con la maestría que se imparte en la FCS. El ingreso a esta maestría sí está restringido a un máximo de 35 por año, cifra que no satisface la demanda bruta ya que a las convocatorias, que se realizan cada dos años, se presenta un número superior y, por lo tanto, se realiza un proceso de selección. De todas formas el número de titulados es particularmente reducido ya que a diciembre de 2011 se habían entregado tan sólo 35 títulos de Magíster en Ciencia Política. Si bien la restricción del ingreso y el nivel superior de los estudios deberían operar a favor de una demanda más calificada, las tasas de egreso son similares a las de la licenciatura. En ese sentido la gratuidad de los estudios unida a una mayor exigencia para estudiantes que suelen trabajar y estudiar al mismo tiempo, parecen operar como desestímulo para la culminación de los estudios. Por otra parte, una proporción importante de los egresados de la licenciatura que tienen vocación académica, buscan realizar estudios de posgrado en el exterior, ya sea porque buscan mejores niveles de formación o la obtención de becas que en Uruguay son prácticamente inexistentes. Un estudio reciente, que ubicó politólogos rioplatenses que se desempeñan en el exterior, muestra que los uruguayos, consultados por las razones que los habían motivado a hacer su doctorado en el exterior, concentran abrumadoramente sus respuestas en la ausencia de doctorados en Uruguay y la calidad que ofrecen las instituciones extranjeras (Malamud y Freidenberg, 2010).

Junto con la maestría de la FCS habría que mencionar la maestría en Comunicación Política y Gestión de Campañas Electorales que se dicta en la UCUDAL. Esta maestría ya ha realizado dos convocatorias, en 2007 y 2010 con 14 y 9 inscriptos respectivamente, y ya cuenta con un titulado.

Finalmente, corresponde mencionar el doctorado en ciencia política, creado en la FCS en 2005 y que a diciembre de 2011 contaba con dos titulados. Se trata de un programa de doctorado basado en la investigación para la tesis, que se apoya en la formación curricular que proporciona la maestría y que ofrece un número limitado de cursos dictados por profesores visitantes. En definitiva, la enseñanza de la ciencia política en Uruguay no está muy diversificada y muestra una fortísima concentración en la UdelaR, cuestión que se explica tanto por la acumulación y concentración académica realizada por el ICP, cuanto por la gratuidad de los estudios de una disciplina que, en principio, no genera mayores expectativas de inserción profesional con ingresos importantes. 


\section{LA PROFESIÓN DE POLITÓLOGO EN URUGUAY}

La creciente masa de titulados en ciencia política, que se puede estimar superó el centenar en el correr del año 2005, generó estímulos para que la profesión adquiriera densidad y se instalara de forma institucionalizada en el medio. Una cuestión relevante al respecto es que en los inicios, la profesión de politólogo no estaba definida y carecía de demanda, a no ser por la que fue generando la propia actividad académica. Pero paralelamente al desarrollo del ICP y el avance de la licenciatura, varios cientistas sociales venían adquiriendo visibilidad pública a través de frecuentes apariciones mediáticas en las que fungían como analistas políticos y, especialmente, difundían y comentaban encuestas de opinión pública (Buquet 2004, Garcé 2005). La difusión de encuestas de intención de voto y luego de otros aspectos de la opinión pública se fue haciendo cada vez más frecuente desde la restauración misma de la democracia. En una primera fase, los medios - de forma plenamente justificadallamaban sociólogos a los especialistas que cumplían esa función, ya que la mayoría de ellos contaba con formación en sociología y muy pocos con formación en ciencia política. Sin embargo, con el correr del tiempo, los medios fueron incorporando y generalizando el término "politólogo" para cualquiera que desarrolle un análisis político y de opinión pública, al punto que se pasó a denominar politólogos a varios sociólogos que terminaron aceptando pacíficamente el mote. Pero para mediados de la década de 1990, fuera de la academia, el análisis político en los medios y los estudios de opinión pública, no existía un espacio profesional al que los politólogos pudieran acceder como tales.

La situación comenzó a mostrar un cambio significativo una década más tarde, cuando comenzó a incluirse el título en ciencia política como requisito en llamados para consultoría y asesoramiento que se realizan desde diversas instituciones públicas. En este aspecto parece haber sido decisivo el acceso del Frente Amplio al gobierno, que, por un lado, no tenía fuertes vínculos con quienes cumplían esas funciones durante los gobiernos anteriores y, por el otro, estaba mucho más próximo al ámbito académico.

Asimismo en el año 2005 comenzó el proceso de fundación de la Asociación Uruguaya de Ciencia Política (AUCiP) que reúne a los profesionales y académicos de la disciplina. Este proceso se desencadenó a partir de un incentivo específico: el cogobierno universitario que incluye representantes de los egresados en los consejos 
de las facultades. En sucesivas instancias en las que correspondía elegir a los representantes del orden, se realizaban reuniones de egresados de la carrera de ciencia política para negociar con los profesionales de las otras carreras y designar candidatos. En ese ámbito se proponía recurrentemente la necesidad de dar continuidad al cuerpo. La iniciativa finalmente fructificó como consecuencia de la elección de autoridades universitarias de 2005. El grupo que se congregó para la ocasión convocó a una serie de asambleas que fueron dando forma a la asociación. Finalmente se convocó el primer congreso uruguayo de ciencia política que en octubre de 2006 fundó oficialmente la AUCiP, aprobó el estatuto y designó sus autoridades. A partir de entonces la asociación ha mantenido un funcionamiento regular realizando un congreso nacional cada dos años y cumpliendo otras actividades.

Entre las diversas actividades emprendidas por AUCiP, una de la mayor importancia ha sido la realización de dos censos que recabaron información sobre los politólogos del Uruguay. De acuerdo al último censo, realizado en $2009^{10}$, sabemos que casi un $70 \%$ de los titulados poseía algún título de posgrado, 13\% doctorados, 43\% maestrías y 10\% diplomas. Asimismo, casi el 60\% de la población censada declaró tener actividad laboral como politólogo o cientista social en al menos un empleo. La probabilidad de tener actividad laboral dentro de la disciplina aumenta significativamente con la titulación alcanzada, de modo que el 90\% de los doctores y casi el 70\% de los magísteres censados declaran que se desempeñan laboralmente como politólogos o cientistas sociales. Por otra parte, cerca de la mitad de quienes se desempeñan dentro de la disciplina lo hacen en el medio académico, mientras que un significativo $20 \%$ trabaja en el ámbito del Poder Ejecutivo y un 10\% lo hace en consultoras de opinión pública, mostrando de esta forma el peso relativo de los distintos ámbitos de inserción profesional de la ciencia política. Asimismo dos tercios de los censados se muestran satisfechos con su situación laboral actual y una cifra muy similar afirma que esa situación ha mejorado respecto a dos años atrás. Esto refleja en un período reciente tanto incrementos salariales para quienes ya se desempeñaban dentro de la disciplina cuanto la apertura de nuevas opciones para los profesionales de la ciencia política.

10 Disponible en www.aucip.org.uy 


\section{LA PRODUCCIÓN ACADÉMICA DE LA CIENCIA POLÍTICA URUGUAYA}

Esta sección se propone informar sobre la producción de la ciencia política uruguaya en términos sistemáticos. Aunque más arriba se ha hecho referencia a los temas y estilos de la investigación politológica, aquí se mostrarán datos e indicadores que refieren a la formación, actividad y producción de investigación de los académicos que se desempeñan en Uruguay. Para ello se utilizarán los CV de los investigadores que pertenecen al Sistema Nacional de Investigadores (SNI) que declararon a la ciencia política como disciplina principal y la base de datos que contiene las publicaciones de dichos investigadores ${ }^{11}$. El SNI se creó en el año 2007 como parte de la política en materia de ciencia y tecnología que promueve el gobierno del Frente Amplio ${ }^{12}$ y tiene como objetivo "fortalecer, expandir y consolidar la comunidad científica nacional, acompañado de la tarea de categorizar y evaluar periódicamente a todos los investigadores, estableciendo un sistema de incentivos económicos" (www.sni. org.uy). Su reglamentación tomó bastante tiempo hasta que en 2008 se realizó la primera convocatoria y en marzo de 2009 se incorporó al primer grupo de investigadores al sistema. Si bien el sistema no incluye a la totalidad de la comunidad académica, porque algunos investigadores no se postularon y otros fueron rechazados, prácticamente todos han procurado su inclusión, ya sea por el prestigio que implica semejante reconocimiento o, simplemente, para acceder al incentivo económico que se otorga a sus integrantes. En consecuencia, la condición de investigador activo en el SNI es un criterio útil y sólido para delimitar el universo de los investigadores nacionales en ciencia política, porque, aunque haya alguna exclusión, seguramente no hay ninguna inclusión injustificada.

La información procesada para este análisis corresponde a los 39 investigadores en ciencia política que figuraban como activos en el SNI al 31/12/11. El primer dato a comentar es el papel central del ICP dentro de este grupo, como ya se ha mencionado. De los 39 investigadores, 32 pertenecen a la UdelaR y 31 al ICP, esto es, el $80 \%$ de los investigadores en ciencia política se desempeña en el ICP, lo que reafirma el rol cuasi hegemónico del Instituto en la materia. Por otra parte, hay tres investigadores de la Ucudal y otros tres de Universidad de Montevideo, mostrando

11 Los CV están disponibles en www.sni.org.uy.

12 El SNI ha sido promovido por investigadores científicos del país desde la década de 1990 y no es la primera inciativa en la materia, ya que en 1999 se creó el Fondo Nacional de Investigadores (FNI), con motivaciones similares aunque sin el nivel de sistematización que tiene el actual. El funcionamiento del FNI fue decayendo como consecuencia de la crisis de 2002 hasta que el gobierno actual lo sustituyó por el SNI. 
que se trata de los dos centros privados desde donde también se desarrolla investigación en ciencia política. Finalmente, llama la atención que sólo una investigadora del CLAEH está incluida en el sistema, siendo que este centro cultiva desde hace décadas temáticas vinculadas a la ciencia política. También la primacía del ICP es absoluta en cuanto al nivel en el que el SNI clasifica a los investigadores, ya que la totalidad de investigadores de nivel III y II pertenece al ICP, constatando así la acumulación de largo plazo realizada por el ICP en contraste con el carácter más incipiente del cultivo de la disciplina en los centros privados.

Un segundo aspecto a relevar es la formación con la que cuenta la comunidad politológica nacional. De los 39 investigadores, 22 cuentan con título de doctorado y 13 con título de maestría. Por otra parte, casi todos los que no tienen título de doctorado (que a su vez, en su amplia mayoría, están en la categoría de candidatos a investigador del SNI) están haciéndolo, a tono con la evolución de las exigencias que se han normalizado en la última década. En cuanto a los doctores, la gran mayoría obtuvo su título en el exterior (18 de 22), la mayoría en América Latina (seis en Brasil, dos en Argentina y uno en México) y el resto en Europa y Estados Unidos (cuatro en USA, dos en Alemania, uno en Francia, uno en España y una en Inglaterra). Esto obedece fundamentalmente a la carencia de estudios doctorales en Uruguay. De los cuatro titulados en el país, dos lo hicieron en un programa especial de Ucudal que ya no se realiza y los otros dos son los primeros y únicos egresados del programa de doctorado de la FCS/UdelaR. Por otra parte, vale la pena destacar que de los cuatro doctorados en USA, sólo uno se desempeña en el ICP, mientras que los otros tres lo hacen en universidades privadas desde hace relativamente poco tiempo, mostrando la apuesta de dichas instituciones por captar uruguayos formados en USA, que en términos generales, han sido difíciles de repatriar (Malamud y Freindenberg, 2010). Finalmente, hay que mencionar que la titulación de maestría muestra un patrón inverso por países, ya que la mayoría de los que obtuvieron dicha titulación lo hicieron en Uruguay (ocho de trece).

Por último interesa observar algunas características de la producción de los investigadores nacionales en ciencia política, tanto en volumen y tipos de productos cuanto por su impacto. Con respecto al volumen de producción se han relevado las publicaciones reportadas por los investigadores en el CV tanto de ciencia política cuanto de sociología y economía, para manejar parámetros de comparabilidad. Asimismo, la producción relevada está diferenciada de acuerdo a diferentes tipos de publicación. Por un lado, los artículos en revistas científicas se subdividen por arbitraje e indexación y, por otra, los libros y capítulos de libros se subdividieron 
de acuerdo al país de la editorial. En cuanto a las revistas, el carácter arbitrado de la publicación lo establece el propio investigador, pero la indexación de la revista en alguno de los tres índices que adoptó el SNI (Scopus, ISI/Thompson y Latindex) la reporta el sistema de forma automática, a partir del nombre de la revista ingresada por el investigador.

La importancia de diferenciar los artículos de acuerdo al arbitraje y la indexación de las revistas deviene de los estándares de evaluación académica que se han venido imponiendo y que ubican al arbitraje por pares como un requisito prácticamente imprescindible para establecer la calidad del producto. Por su parte, la diferenciación de la publicación de capítulos y libro según el país de la editorial responde al supuesto general de que la publicación en el exterior, particularmente en un país pequeño como Uruguay, da cuenta de un grado mayor de exigencia, ya sea directamente sobre la calidad del producto o indirectamente a través de la selección de los autores por parte de redes internacionales de investigación. En definitiva se asume que tanto la publicación de artículos en revistas arbitradas y, especialmente indexadas, cuanto de capítulos y libros en el exterior, representan producción de mayor calidad. Al culminar esta sección se intentará demostrar, a través del impacto de la producción de los investigadores la validez de estos supuestos. En primer término veamos el volumen de la producción en ciencia política en comparación con las otras disciplinas sociales ${ }^{13}$.

13 En este caso la información corresponde a todos los investigadores de las tres disciplinas que alguna vez fueron categorizados por el SNI como activos, por lo que el total de los que aparecen en ciencia política son 46 y no 39 como en el resto de los análisis que se realizan. 


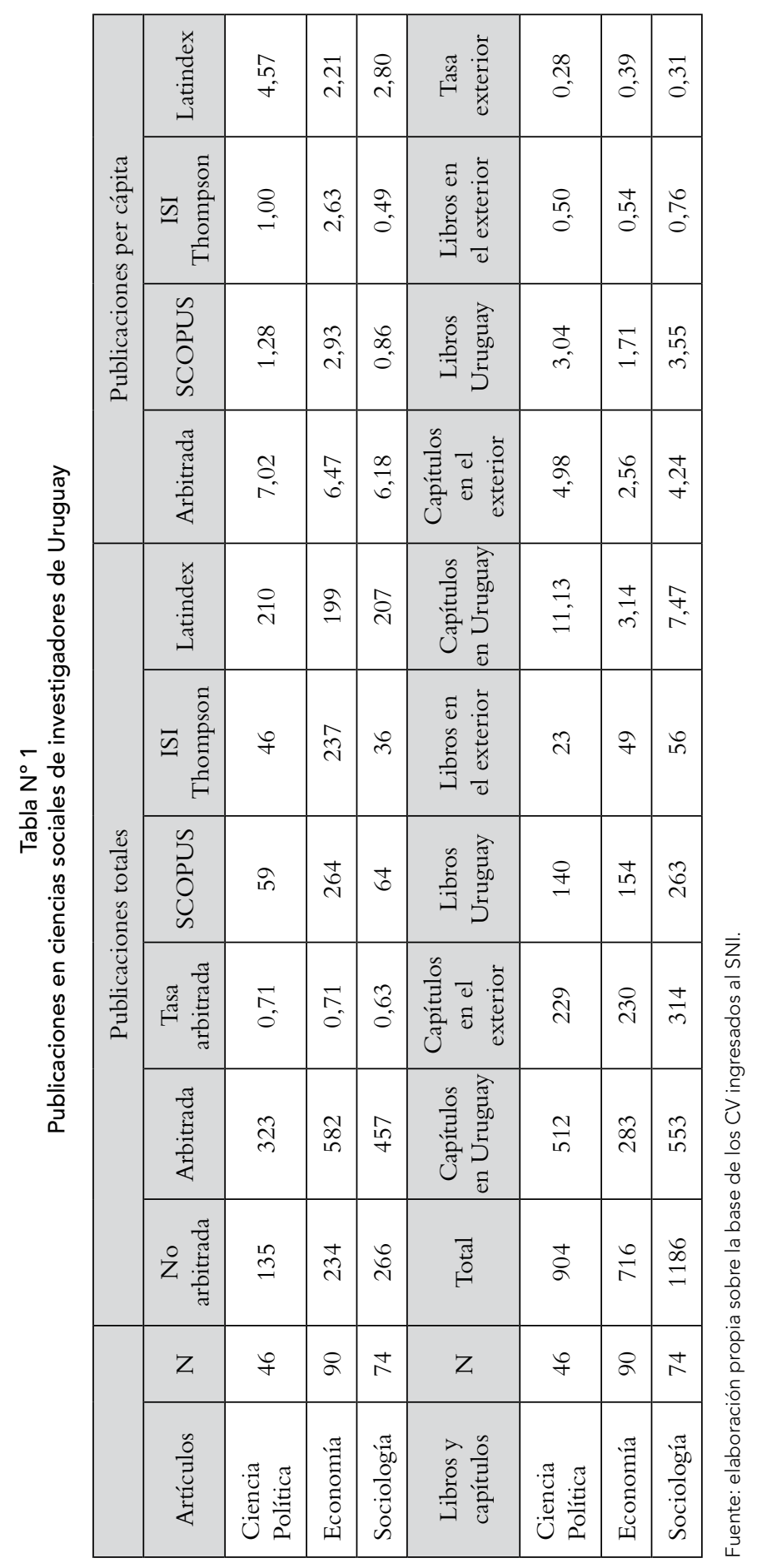


Lo primero que se observa es que el total de artículos publicados por politólogos es inferior a los publicados por sociólogos y economistas, pero los politólogos han publicado más capítulos de libros que los economistas, aunque menos que los sociólogos. Estos datos ya muestran una impronta que caracteriza tanto a la ciencia política como a la sociología, que es la mayor propensión a utilizar el formato de capítulo de libro para difundir su producción que los economistas. En cuanto a la publicación en revistas, las tres disciplinas muestran una tasa de arbitraje próxima a los dos tercios, aunque en ciencia política y economía es algo superior a sociología. Por su parte, en cuanto a la indexación, la ciencia política y la sociología muestran una mayor propensión a publicar en revistas incluidas en Latindex, mientras que los economistas destinan mayoritariamente su producción a revistas indexadas en Scopus o ISI. Finalmente, en cuanto a libros y capítulos la ciencia política muestra la menor propensión a publicar en el exterior, con cifras muy similares a las de sociología e inferiores a las de economía.

De todas formas los datos de producción global no resultan muy relevantes por cuanto el tamaño de las respectivas comunidades académicas difiere significativamente. El SNI incluye al doble de economistas y una vez y media de sociólogos que de cientistas políticos. Por lo tanto parece más relevante ver indicadores de producción per cápita. En promedio, un politólogo nacional ha publicado siete artículos en revistas arbitradas, once capítulos de libro en Uruguay, cinco capítulos de libro en el exterior, tres libros en Uruguay y medio libro en el exterior. En este campo la tasa de publicación per cápita en revistas arbitradas de los politólogos es superior a la de los economistas y los sociólogos, aunque esa producción está concentrada en revistas del catálogo Latindex. En cambio, los economistas son los que publican más artículos per cápita en revistas indexadas en ISI y Scopus. Pero la publicación per cápita de artículos en revistas arbitradas de los politólogos supera a la de los sociólogos en todos los rubros. La publicación de capítulos de libro per cápita de los politólogos también es superior a la que realizan economistas y sociólogos, tanto en el país como en el exterior. En cambio, los sociólogos publican más libros per cápita que los politólogos, tanto en Uruguay como el exterior y, si bien los politólogos publican mayor cantidad de libros per cápita que los economistas, estos últimos publican algo más que los politólogos en el exterior.

Pasemos ahora a evaluar más detenidamente la producción de los cientistas políticos en particular. Una primera diferenciación es la que resulta de observar los tipos de publicaciones de acuerdo al nivel otorgado por SNI a los investigadores. Como era de esperar, la cantidad y calidad de la producción son crecientes en función del nivel en el SNI. 


\section{Gráfico $N^{\circ} 2$}

Publicaciones per cápita según tipo de publicación y nivel SNI

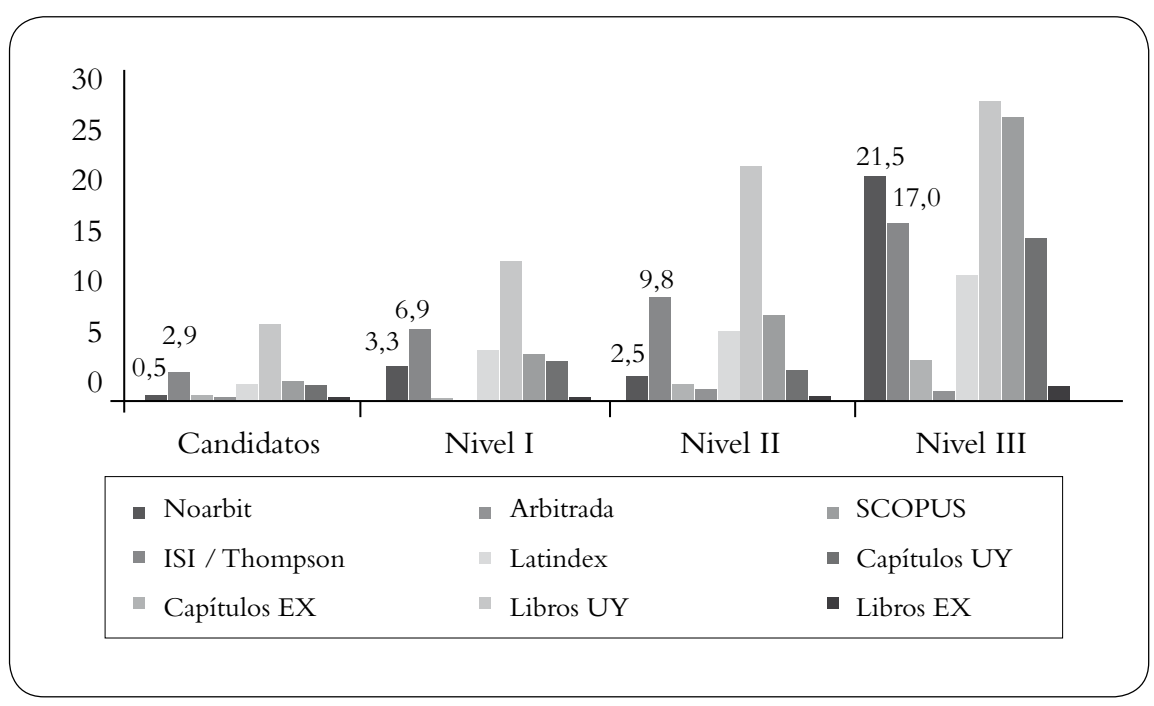

Fuente: elaboración propia sobre la base de los CV ingresados al SNI.

El gráfico muestra claramente que el volumen de las publicaciones en la mayoría de las modalidades crece de acuerdo con el nivel. Tanto los artículos en revistas arbitradas como los capítulos publicados en el país muestran un patrón de incremento sostenido sin saltos importantes que evidentemente responden al avance de la carrera académica de los investigadores. Por su parte, el promedio de capítulos publicados en el exterior, si bien también aumenta según el nivel, claramente da un salto en el nivel III, donde la cifra más que triplica al promedio del nivel II. Asimismo, los investigadores de nivel III son los únicos que publican más capítulos en el exterior que artículos en revistas arbitradas. Esto muestra, por un lado, que la posibilidad de publicar capítulos en el exterior está fuertemente asociada a la consolidación de la carrera académica y al prestigio internacional de los investigadores y, por otro, que la publicación de artículos en revistas arbitradas constituye una práctica más reciente dentro de la comunidad académica.

Un segundo aspecto a observar es la evolución en el tiempo de los tipos de publicación y su peso relativo en el total de la producción académica. 
Gráfico $N^{\circ} 3$

Tipos de publicación de los politólogos de Uruguay por periodos.

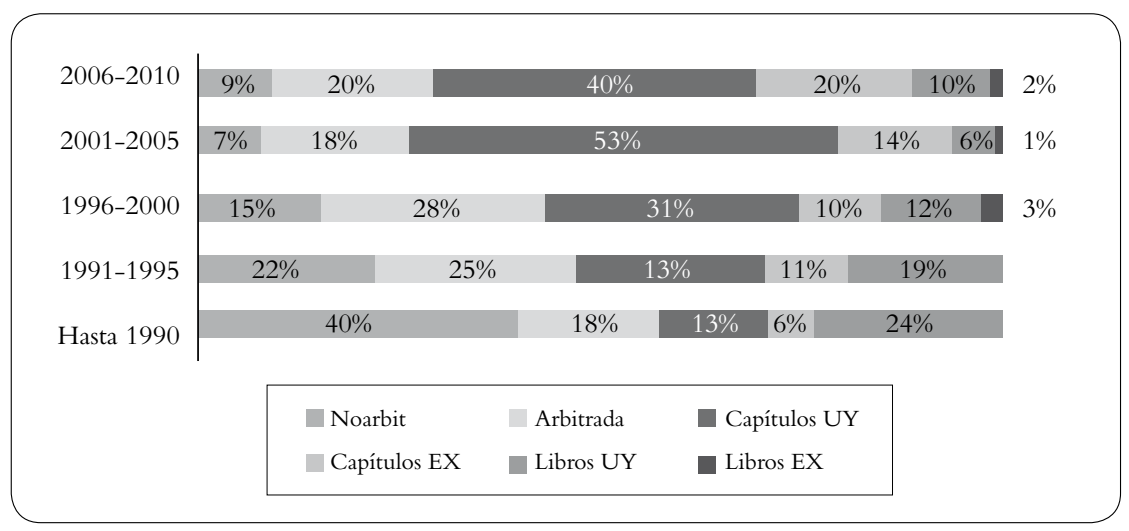

Fuente: elaboración propia sobre la base de los CV ingresados al SNI.

El gráfico permite apreciar claramente cómo dos tipos de publicación han ido perdiendo peso con el tiempo mientras que otros tres se han incrementado. En los albores de la ciencia política uruguaya la producción se concentraba en la publicación de libros nacionales y artículos en revistas no arbitradas que alcanzaban a casi los dos tercios de la producción. En la última década, en cambio, los artículos en revistas arbitradas y los capítulos de libro publicados en Uruguay y el exterior superan el $80 \%$ de la producción. A su vez, los últimos cinco años parecen mostrar una tendencia al incremento de la publicación de capítulos en el exterior en desmedro de los publicados en el país. Esto reafirmaría la idea de que tanto los artículos en revistas arbitradas (particularmente las indexadas) como los capítulos de libro publicados en el exterior se van convirtiendo en el producto típico de la ciencia política uruguaya que aspira a lograr niveles internacionales de calidad.

Pero para tener una imagen más precisa de la calidad de las publicaciones de la ciencia política uruguaya, veamos por último el impacto que ha tenido esa producción. Una forma crecientemente generalizada de aproximarse a la calidad de la producción científica es observar el impacto en citas que genera. Con este criterio, una reciente publicación de Altman (2011) elabora un ranking de departamentos de ciencia política en América Latina. El estudio no resulta alentador para el Uruguay ya que ubica al ICP en el lugar 19 entre los 21 departamentos considerados. Pero el trabajo de Altman es muy restrictivo con respecto a la fuente de información que utiliza; sólo considera los artículos indexados en la Web of Knowledge (WoK, ex ISI) y las citas recibidas por ellos. 
Si bien es cierto que la indexación ISI o SCOPUS se han convertido en estándares de calidad para las ciencia sociales en el primer mundo, está claro que hasta ahora en Uruguay no existe ningún incentivo específico para publicar en revistas indexadas en esos ámbitos, no se ha generado el hábito de hacerlo y, el hecho de que la gran mayoría de las revistas que figuran en esas bases publiquen artículos exclusivamente en inglés, constituye un obstáculo evidente para colocar la producción de nuestros investigadores. De todas formas, una búsqueda realizada a mediados de 2011 localizó 180 citas en WoK a publicaciones de los investigadores del ICP aunque no hayan sido publicados en revistas indexadas por ellos (Buquet, 2011). Estos datos representan un muy significativo aumento con relación a las 65 citas reportadas por Garcé (2005). Más allá de que parte de la explicación de este avance puede obedecer a la incorporación a WoK de revistas en español que en 2005 no estaban incluidas, es indiscutible que el incremento de las cifras da cuenta de un fuerte aumento de la visibilidad de la producción de la ciencia política nacional.

Por otra parte, es posible ir más allá del restringido ámbito de WoK, ya que desde hace algunos años es posible ubicar citas a trabajos científicos en un entorno más amplio y, en cierto sentido, más democrático: el google académico ${ }^{14}$. Si bien las citas que se obtienen en el google académico no cumplen una serie de requisitos deseables que se establecen en otros ámbitos, también ofrece algunas ventajas, la más importante de ellas consistente en ampliar el campo de observación del impacto de la producción, particularmente en el área de habla hispana y de América Latina, cuyo acceso a WoK es limitado, y que deberían ser de especial interés para nuestra academia. Por otra parte, no es de esperar que surjan diferencias importantes en cuanto a las consecuencias de utilizar la base de WoK o la de google académico, ya que existe una correlación estadísticamente significativa entre el índice $\mathrm{H}$ basado en google acholar y número de entradas en WoK (Altman, 2011) o entre el número de citas obtenidas en google académico y las ubicadas en WoK (Buquet, 2011), por lo que la información agregada puede considerarse completamente consistente.

Los 39 investigadores en ciencia política categorizados en el SNI obtienen en conjunto más de 5000 citas en google académico, lo que de por sí da cuenta de un impacto relevante de 130 citas per cápita. De todas formas existe una considerable concentración de las citas, ya que sólo ocho investigadores con más de 200 citas cada uno, concentran el 75\% del total. En consecuencia, más que el total de citas recibi-

14 Las citas y los índices de impacto que se utilizan en este estudio se obtuvieron a través del programa Publish or Perish (Ver Harzing 2007). 
das por los politólogos uruguayos, lo que interesa saber es qué factores de la carrera académica determinan el impacto de la producción de un cientista político. Para determinar este aspecto se realizaron una serie de regresiones con la base de datos de las publicaciones y otra información ingresadas en los CV del SNI. Como variable dependiente se optó por utilizar un promedio de los índices H y G que calcula el programa Publish or Perish, aunque otras opciones, como cada uno de esos índices por separado o el total de citas, arrojan resultados muy similares. Aquí se presentan algunos modelos que, en todos los casos, tienen alta significación estadística y explican del orden del $80 \%$ de la varianza.

Tabla $\mathrm{N}^{\circ} 2$

Regresiones para explicar el impacto de los investigadores uruguayos

\begin{tabular}{|l|c|c|c|c|}
\hline \multirow{2}{*}{ Variables } & \multicolumn{4}{|c|}{ Modelos } \\
\cline { 2 - 5 } & 1 & 2 & 3 & 4 \\
\hline Nivel del SNI & $1,02^{\star \star}$ & & & \\
\hline Título obtenido &,- 058 & & & \\
\hline País de obtención &,- 100 & & & \\
\hline Año primera publicación &, 107 & & & \\
\hline Artículos en revistas arbitradas & & $0,331 \star$ & & \\
\hline Artículos indexados en SCOPUS & & $0,581^{\star \star}$ & & $0,228 \star \star$ \\
\hline Artículos indexados en ISI & & $-0,297 \star$ & & \\
\hline Artículos indexados en Latindex & & $0,310^{\star}$ & & $0,350 \star \star$ \\
\hline Capítulos publicados en Uruguay & & & $0,414 \star \star$ & \\
\hline Capítulos publicados en el exterior & & & $0,785 \star \star$ & \\
\hline Libros publicados en Uruguay & & &,- 149 & \\
\hline Libros publicados en el exterior & & & $-0,197 \star$ & \\
\hline Total de capítulos publicados & & & & $0,507 \star \star$ \\
\hline R2 &, 786 &, 806 &, 826 &, 871 \\
\hline
\end{tabular}

a. Dependent Variable: PromedioHG

Fuente: Elaboración propia sobre la base de los CV del SNI.

El primer modelo incluye como variables independientes: el nivel que el SNI otorgó a los investigadores, el máximo título alcanzado ( $1=$ licenciatura; $2=$ maestría y $3=$ doctorado), el país donde se obtuvo dicho título (1=Uruguay; $2=$ América Latina y $3=$ Primer Mundo) y el año de la primera publicación. El nivel del SNI debería 
estar asociado positivamente con el impacto; si bien en el SNI no se utilizan explícitamente indicadores de impacto para categorizar a los investigadores, el principal criterio consiste en determinar la relevancia de la producción, donde podemos suponer que el impacto está implícito. También deberíamos esperar que cuanto mayor sea el nivel del título alcanzado, los investigadores generen mayor impacto. Asimismo se asume que la formación en el exterior y, especialmente en el primer mundo, dada la mayor calidad que se le atribuye, debería influir positivamente en el impacto de la producción de los investigadores. Finalmente, el año de la primera publicación es un proxy de la extensión de la carrera académica (con signo inverso), por lo que deberíamos pensar que carreras más extensas generan mayor impacto.

En primer término se observa que el nivel en el SNI, como podía esperarse, está significativamente asociado al impacto de los investigadores pero, ni el nivel de estudios alcanzado ni el país donde se realizaron esos estudios muestra relación con el impacto de la producción. Tampoco aparece significativamente asociada con el impacto la extensión de la trayectoria académica y, además, en los tres casos los signos de los coeficientes son los opuestos a los esperados.

En cuanto al efecto de las publicaciones, no sólo el total, sino diversos tipos están significativamente asociados con el impacto que han producido los investigadores (modelos 2 y 3). Así, los artículos arbitrados en general y los indexados en Scopus y Latindex en particular, se asocian positivamente al impacto. Sin embargo, ocurre lo contrario con los artículos publicados en ISI, que aunque asociados significativamente con el impacto, lo hacen en sentido inverso (el coeficiente tiene signo negativo). Esto evidentemente es producto de la escasa presencia en ese ámbito de la producción politológica uruguaya. Asimismo, la publicación de capítulos, tanto en Uruguay como en el exterior, muestra una fuerte asociación con el impacto. En cambio, la publicación de libros en Uruguay no tiene significación estadística y la publicación de libros en el exterior está asociada al impacto, pero, con signo contrario al esperado. Finalmente, el modelo 4 pretende rescatar los tipos de publicación que parecen explicar la mayor parte del impacto; se trata de los artículos en revistas indexadas, tanto en Scopus como en Latindex, así como el total de capítulos de libro publicados. El modelo con estas tres variables explica casi el 90\% de la varianza. Como se mostró más arriba, este tipo de publicaciones se han convertido en el destino claramente predominante de la producción de la ciencia política nacional y, por lo visto, semejante opción viene rindiendo frutos en materia de impacto. 


\section{A MODO DE SÍNTESIS}

La ciencia política en Uruguay ha tenido un desarrollo tardío, tanto con relación al mundo cuanto con respecto a los países más importantes de América Latina. Su instalación como disciplina se concretó recién después de la última transición a la democracia, a partir de 1985. Pero desde ese momento en adelante ha desarrollado un espacio y un prestigio creciente. En la actualidad se puede hablar de una disciplina consolidada, tanto en el espacio académico como en el ámbito profesional. En este proceso ha jugado un rol central el ICP, que concentra al 80\% de los investigadores de la disciplina y un porcentaje aún mayor de la formación correspondiente. Partiendo de un estilo teórico y ensayístico y utilizando abordajes parroquiales para sus objetos de estudio, el trabajo académico ha ido incorporando crecientemente metodologías más sofisticadas, ha ampliado su horizonte comparativo y ha ido incorporando estándares de calidad que se aproximan a los utilizados internacionalmente.

La ciencia política uruguaya cuenta con un plantel de investigadores que ostenta buenos niveles de formación y es productivo en términos generales, tanto en cantidad como en calidad. Si bien en términos internacionales, incluso regionales, no podemos estar satisfechos, la producción politológica nacional puede considerase aceptable. Pero evidentemente debe reforzarse la dirección ascendente que parece insinuar los datos referidos a los últimos años y apostar a una inserción aún más fuerte en el contexto regional. En este sentido, la política de promoción de la investigación en ciencia política debería reforzar los incentivos para promover la publicación de artículos en revistas arbitradas (especialmente indexadas) y/o de capítulos en libros internacionales que son los tipos de publicación que parecen generar un mayor impacto.

\section{Referencias Bibliográficas}

Altman, David (2011). "Where is Knowledge Generated? On the Productivity and Impact of Political Science Departments in Latin America". European Political Science Vol. 11, $\mathrm{N}^{\circ}$, pp. 71-87.

Altman, David (2005). "La institucionalización de la Ciencia Política en Chile y América Latina: Una mirada desde el sur”. Revista de Ciencia Política Vol. 25, Nº1, pp. 3-15.

De Sierra, Gerónimo (2005). "Social Sciences in Uruguay". Social Science Information Vol. 44, $\mathrm{N}^{\circ} 2-3$, pp. 473-520.

Buquet, Daniel (2011). Informe de Investigación (2005-2010). Instituto de Ciencia Política, FCS/UdelaR (inédito). 
Chasquetti, Daniel (2010). "El lento camino de la política comparada en Uruguay”, en Diego Gantus (comp.) La enseñanza de la Ciencia Política en las universidades de América Latina. Rosario: Editorial Ciudad Gótica.

Garcé, Adolfo (2005). "La ciencia política en Uruguay: un desarrollo tardío, intenso y asimétrico". Revista de Ciencia Política,Vol. 25, No 1, pp. 232-244.

Harzing, A.W. (2007). Publish or Perish, disponible en http://www.harzing.com/pop.htm

Caetano, Gerardo; Rilla, José y Pérez, Romeo (1987). "La partidocracia uruguaya. Historia y teoría de la centralidad de los partidos políticos". Cuadernos del CLAEH No 44.

Caetano, Gerardo, José Rilla, Pablo Mieres y Pérez, Romeo (1992). Partidos y Electores: centralidad $y$ cambios. Montevideo: Ediciones de la Banda Oriental.

Instituto de Ciencia Política (1989). Los Partidos Políticos de Cara al 90. Montevideo, Fundación de Cultura Universitaria.

Instituto de Ciencia Política (org) (2002). Carlos Real de Azúa, pionero de la ciencia política en Uruguay (1916-1977): Evocación a 25 años de su desaparición física (Mesa Redonda). Montevideo: Cámara de Representantes.

Instituto de Historia de las Ideas (2007). "In memoriam: Arturo Ardao (1912-2003), Julio Barreiro (1922-2005), Carlos Mato (1932-2003)”. Cuadernos de Historia de las Ideas.Vol. 8: 15-21.

Malamud, Andrés y Freidenberg, Flavia (2010). "La diáspora rioplatense: Presencia e impacto de los politólogos argentinos, brasileños y uruguayos en el exterior". Trabajo presentado en el VIII Congreso Iberoamericano de Indicadores de Ciencia y Tecnología. Red Iberoamericana de Indicadores de Ciencia y Tecnología (RICYT), Observatorio Iberoamericano de la Ciencia, la Tecnología y la Sociedad (OEI), Instituto de Estudios Documentales sobre Ciencia y Tecnología (IEDCyT-CSIC), Madrid, 5 y 6 de octubre.

Pérez Antón, Romeo (1992). “Ciencia Política”, en Caetano, Gerardo, José Rilla, Pablo Mieres y Pérez, Romeo (1992). Partidos y Electores: centralidad y cambios. Montevideo: Ediciones de la Banda Oriental.

Real de Azúa, Carlos (1988). Partidos, Política y Poder en el Uruguay. Montevideo: Facultad de Humanidades y Ciencias.

Rocha, Cecilia (2012). El desarrollo de la Ciencia Política en Uruguay (1987-2009): Temas, teorías y metodología. Monografía final de la Licenciatura en Ciencia Política. Montevideo: Facultad de Ciencias Sociales.

Recibido: 24 abril 2012 / Aprobado: 20 junio 2012 